\title{
Literature-based discovery of diabetes- and ROS-related targets
}

\author{
Junguk Hur ${ }^{1,2}$, Kelli A Sullivan², Adam D Schuyler ${ }^{4}$, Yu Hong ${ }^{2}$, Manjusha Pande ${ }^{2,3}$, David J States ${ }^{5}$, H V Jagadish ${ }^{1,3}$, \\ Eva L Feldman ${ }^{1,2,3^{*}}$
}

\begin{abstract}
Background: Reactive oxygen species (ROS) are known mediators of cellular damage in multiple diseases including diabetic complications. Despite its importance, no comprehensive database is currently available for the genes associated with ROS.

Methods: We present ROS- and diabetes-related targets (genes/proteins) collected from the biomedical literature through a text mining technology. A web-based literature mining tool, SciMiner, was applied to 1,154 biomedical papers indexed with diabetes and ROS by PubMed to identify relevant targets. Over-represented targets in the ROS-diabetes literature were obtained through comparisons against randomly selected literature. The expression levels of nine genes, selected from the top ranked ROS-diabetes set, were measured in the dorsal root ganglia (DRG) of diabetic and non-diabetic DBA/2J mice in order to evaluate the biological relevance of literature-derived targets in the pathogenesis of diabetic neuropathy.
\end{abstract}

Results: SciMiner identified 1,026 ROS- and diabetes-related targets from the 1,154 biomedical papers (http://jdrf. neurology.med.umich.edu/ROSDiabetes/). Fifty-three targets were significantly over-represented in the ROS-diabetes literature compared to randomly selected literature. These over-represented targets included well-known members of the oxidative stress response including catalase, the NADPH oxidase family, and the superoxide dismutase family of proteins. Eight of the nine selected genes exhibited significant differential expression between diabetic and nondiabetic mice. For six genes, the direction of expression change in diabetes paralleled enhanced oxidative stress in the DRG.

Conclusions: Literature mining compiled ROS-diabetes related targets from the biomedical literature and led us to evaluate the biological relevance of selected targets in the pathogenesis of diabetic neuropathy.

\section{Background}

Diabetes is a metabolic disease in which the body does not produce or properly respond to insulin, a hormone required to convert carbohydrates into energy for daily life. According to the American Diabetes Association, 23.6 million children and adults, approximately $7.8 \%$ of the population in the United States, have diabetes [1]. The cost of diabetes in 2007 was estimated to be $\$ 174$ billion [1]. The micro- and macro-vascular complications of diabetes are the most common causes of renal failure, blindness and amputations leading to significant mortality, morbidity and poor quality of life; however,

\footnotetext{
* Correspondence: efeldman@umich.edu

'Bioinformatics Program, University of Michigan, Ann Arbor, MI 48109, USA
}

Full list of author information is available at the end of the article incomplete understanding of the causes of diabetic complications hinders the development of mechanism-based therapies.

In vivo and in vitro experiments implicate a number of enzymatic and non-enzymatic metabolic pathways in the initiation and progression of diabetic complications [2] including: (1) increased polyol pathway activity leading to sorbitol and fructose accumulation, $\mathrm{NAD}(\mathrm{P})$-redox imbalances and changes in signal transduction; (2) nonenzymatic glycation of proteins yielding "advanced glycation end-products" (AGEs); (3) activation of protein kinase $\mathrm{C}$ (PKC), initiating a cascade of intracellular stress responses; and (4) increased hexosamine pathway flux $[2,3]$. Only recently has a link among these pathways been established that provides a unified mechanism of tissue damage. Each of these pathways directly 
and indirectly leads to overproduction of reactive oxygen species (ROS) [2,3].

ROS are highly reactive ions or small molecules including oxygen ions, free radicals and peroxides, formed as natural byproducts of cellular energy metabolism. ROS are implicated in multiple cellular pathways such as mitogen-activated protein kinase (MAPK) signaling, c-Jun amino-terminal kinase (JNK), cell proliferation and apoptosis [4-6]. Due to the highly reactive properties of ROS, excessive ROS may cause significant damage to proteins, DNA, RNA and lipids. All cells express enzymes capable of neutralizing ROS. In addition to the maintenance of antioxidant systems such as glutathione and thioredoxins, primary sensory neurons express two main detoxifying enzymes: superoxide dismutase (SOD) [7] and catalase [8]. SOD converts superoxide $\left(\mathrm{O}_{2^{-}}\right)$to $\mathrm{H}_{2} \mathrm{O}_{2}$, which is reduced to $\mathrm{H}_{2} \mathrm{O}$ by glutathione and catalase [8]. SOD1 is the main form of SOD in the cytoplasm; SOD2 is located within the mitochondria. In neurons, SOD1 activity represents approximately $90 \%$ of total SOD activity and SOD2 approximately 10\% [9]. Under diabetic conditions, this protective mechanism is overwhelmed due to the substantial increase in ROS, leading to cellular damage and dysfunction [10].

The idea that increased ROS and oxidative stress contribute to the pathogenesis of diabetic complications has led scientists to investigate different oxidative stress pathways $[7,11]$. Inhibition of ROS or maintenance of euglycemia restores metabolic and vascular imbalances and blocks both the initiation and progression of complications [12,13]. Despite the significant implications and extensive research into the role of ROS in diabetes, no comprehensive database regarding ROS-related genes or proteins is currently available.

In the present study, a comprehensive list of ROSand diabetes-related targets (genes/proteins) was compiled from the biomedical literature through text mining technology. SciMiner, a web-based literature mining tool [14], was used to retrieve and process documents and identify targets from the text. SciMiner provides a convenient web-based platform for target-identification within the biomedical literature, similar to other tools including EBIMed [15], ALI BABA [16], and PolySearch [17]; however, SciMiner is unique in that it searches full text documents, supports free-text PubMed query style, and allows the comparison of target lists from multiple queries.

The ROS-diabetes targets collected by SciMiner were further tested against randomly selected non-ROS-diabetes literature to identify targets that are significantly overrepresented in the ROS-diabetes literature. Functional enrichment analyses were performed on these targets to identify significantly over-represented biological functions in terms of Gene Ontology (GO) terms and pathways.
In order to confirm the biological relevance of the over-represented ROS-diabetes targets, the gene expression levels of nine selected targets were measured in dorsal root ganglia (DRG) from mice with and without diabetes. DRG contain primary sensory neurons that relay information from the periphery to the central nervous system (CNS) $[7,10,18]$. Unlike the CNS, DRG are not protected by a blood-nerve barrier, and are consequently vulnerable to metabolic and toxic injury [19]. We hypothesize that differential expression of identified targets in DRG would confirm their involvement in the pathogenesis of diabetic neuropathy.

\section{Methods}

\section{Defining ROS-diabetes literature}

To retrieve the list of biomedical literature associated with ROS and diabetes, PubMed was queried using ("Reactive Oxygen Species"[MeSH] AND "Diabetes Mellitus"[MeSH]). This query yielded 1,154 articles as of April 27, 2009. SciMiner, a web-based literature mining tool [14], was used to retrieve and process the abstracts and available full text documents to identify targets (full text documents were available for approximately $40 \%$ of the 1,154 articles). SciMiner-identified targets, reported in the form of HGNC [HUGO (Human Genome Organization) Gene Nomenclature Committee] genes, were confirmed by manual review of the text.

\section{Comparison with human curated data (NCBI Gene2PubMed)}

The NCBI Gene database provides links between Gene and PubMed. The links are the result of (1) manual curation within the NCBI via literature analysis as part of generating a Gene record, (2) integration of information from other public databases, and (3) GeneRIF (Gene Reference Into Function) in which human experts provide a brief summary of gene functions and make the connections between citation (PubMed) and Gene databases. For the 1,154 ROS-diabetes articles, genepaper associations were retrieved from the NCBI Gene database. Non-human genes were mapped to homologous human genes through the NCBI HomoloGene database. The retrieved genes were compared against the SciMiner derived targets. Any genes missed by SciMiner were added to the ROS-diabetes target set.

\section{Protein-protein interactions among ROS-diabetes targets}

To indirectly examine the association of literature derived targets (by SciMiner and NCBI Gene2PubMed) with ROS and diabetes, protein-protein interactions (PPIs) among the targets were surveyed. This was based on an assumption that targets are more likely to have PPIs with each other if they are truly associated within the same biological functions/pathways. A PPI network 
of the ROS-diabetes targets was generated using the Michigan Molecular Interactions (MiMI, http://mimi. ncibi.org/) database [20] and compared against 100 PPI networks of randomly drawn sets (the same number of the ROS-diabetes target set) from HUGO. A standard Z-test and one sample T-test were used to calculate the statistical significance of the ROS-diabetes PPI network with respect to the random PPI networks.

\section{Functional enrichment analysis}

Literature derived ROS-diabetes targets (by SciMiner and NCBI Gene2PubMed) were subject to functional enrichment analyses to identify significantly over-represented biological functions in terms of Gene Ontology [21], pathways (Kyoto Encyclopedia of Genes and Genomes (KEGG, http://www.genome.jp/kegg/) [22] and Reactome http://www.reactome.org/[23]). Fisher's exact test [24] was used to calculate the statistical significance of these biological functions with Benjamini-Hochberg $(\mathrm{BH})$ adjusted p-value $<0.05$ [25] as the cut-off.

\section{Over-represented ROS-diabetes targets Defining background corpora}

To identify a subset of targets that are highly overrepresented within the ROS-diabetes targets, the frequency of each target (defined as the number of documents in which the target was identified divided by the number of total documents in the query) was compared against the frequencies in randomly selected background corpora. Depending on how the background set is defined, over-represented targets may vary widely; therefore, to maintain the background corpora close to the ROS and diabetes context, documents were selected from the same journal, volume, and issue of the 1,154 ROS-diabetes documents, but were NOT indexed with "Reactive Oxygen Species"[MeSH] nor "Diabetes Mellitus"[MeSH]. For example, one of the ROS-diabetes articles (PMID: 18227068), was published in the Journal of Biological Chemistry, Volume 283, Issue 16. This issue contained 85 papers, 78 of which were not indexed with either "Reactive Oxygen Species"[MeSH] or "Diabetes Mellitus"[MeSH] indexed. One of these 78 papers was randomly selected as a background document. Three sets of 1,154 documents were selected using this approach and processed using SciMiner. Identified targets were confirmed by manual review for accuracy.

\section{Identifying significantly over-represented targets}

ROS-diabetes targets were tested for over-representation against targets identified from the three background sets. Fisher's exact test was used to determine if the frequency of each target in the ROS-diabetes target set was significantly different from that of the background sets. Any targets with a $\mathrm{BH}$ adjusted $\mathrm{p}$-value $<0.05$ in at least two of the three comparisons were deemed to be an over-represented ROS-diabetes target. Functional enrichment analyses were performed on these overrepresented ROS-diabetes targets as described above.

\section{Selecting targets for real-time RT-PCR}

A subset of targets were selected for RT-PCR from the top 10 over-represented ROS-diabetes targets excluding insulin and NADPH oxidase 5 (NOX5), which does not have a mouse ortholog. Nitric oxide synthase 1 (NOS1), the main generator of nitric oxide, ranked at the $15^{\text {th }}$ position and was additionally selected for inclusion in the test set.

\section{Differential gene expression by real-time RT-PCR Mice}

DBA/2J mice were purchased from the Jackson Laboratory (Bar Harbor, ME). Mice were housed in a pathogen-free environment and cared for following the University of Michigan Committee on the Care and Use of Animals guidelines. Mice were fed AIN76A chow (Research Diets, New Brunswick, NJ). Male mice were used for this study.

\section{Induction of diabetes}

Two treatment groups were defined: control $(\mathrm{n}=4)$ and diabetic $(n=4)$. Diabetes was induced at 13 weeks of age by low-dose streptozotocin (STZ) injections, $50 \mathrm{mg} /$ $\mathrm{kg}$ /day for five consecutive days. All diabetic mice received LinBit sustained release insulin implants (LinShin, Toronto, Canada) at 8 weeks post-STZ treatment. Insulin implants were replaced every 4 weeks, at 12 and 16 weeks post-STZ treatment. At 20 weeks post-STZ treatment, mice were euthanized by sodium pentobarbital overdose and DRG were harvested as previously described [26].

\section{Real-time RT-PCR}

The gene expression of the selected nine literaturederived ROS-diabetes targets in DRG was measured using real-time RT-PCR in duplicate. The amount of mRNA isolated from each DRG was normalized to an endogenous reference [Tbp: TATA box binding protein; $\Delta$ cycle threshold $\left.\left(\mathrm{C}_{\mathrm{T}}\right)\right]$.

\section{Results}

\section{Identification of ROS-diabetes targets}

A total of 1,021 unique targets were identified by SciMiner from the 1,154 ROS-diabetes papers defined by the query of ("Reactive Oxygen Species"[MeSH] AND "Diabetes Mellitus"[MeSH]) and confirmed by manual review. Table 1 contains the top 10 most frequently mentioned targets in the ROS-diabetes papers. Insulin was the most frequently mentioned target, followed by superoxide dismutase 1 and catalase.

The NCBI Gene2PubMed database, containing expertcurated associations between the NCBI Gene and PubMed databases, revealed 90 unique genes associated 
Table 1 Top 10 most frequent ROS-diabetes targets

\begin{tabular}{|c|c|c|c|}
\hline Symbol & Name & \#Paper & Match Strings \\
\hline INS & insulin & 503 & INS | insulin | proinsulin | \\
\hline SOD1 & superoxide dismutase 1 & 368 & Sod1 | SOD1 | SOD1 |* \\
\hline CAT & catalase & 241 & CAT $\mid$ catalase $\mid *$ \\
\hline PRKCA & protein kinase $C$, alpha & 194 & PKCA | PKC-alpha | * \\
\hline ALB & albumin & 179 & albumin | serum albumin | \\
\hline NOX5 & NADPH oxidase 5 & 177 & NOX5 | nadph oxidase | \\
\hline NOS2A & nitric oxide synthase $2 \mathrm{~A}$ & 144 & NOS | iNOS | Nos2 |* \\
\hline $\mathrm{XDH}$ & $\begin{array}{l}\text { xanthine } \\
\text { dehydrogenase }\end{array}$ & 133 & $\begin{array}{r}\text { XOR |xanthine } \\
\text { dehydrogenase| * }\end{array}$ \\
\hline AGT & angiotensinogen & 131 & Ang-II | ANG | AGT | Angl | \\
\hline TNF & tumor necrosis factor & 120 & TNFA | TNF | TNF-alpha | \\
\hline
\end{tabular}

* Matching strings are truncated to fit in the table. The full contents are available in the Additional File 2. '\#Paper' refers to the number of documents in which each target was mentioned at least once.

with the 1,154 ROS-diabetes papers (Additional File 1). SciMiner identified 85 out of these 90 targets, indicating a $94 \%$ recall rate. Five targets missed by SciMiner were added to the initial ROS-diabetes target set to result in 1,026 unique targets (Additional File 2).

\section{PPI network of the ROS-diabetes targets}

The PPI network among the ROS-diabetes targets was evaluated using MiMI interaction data. This was based on the assumption that targets commonly related to a certain topic are more likely to have frequent interactions with each other. One hundred PPI networks were generated for comparison using the same number of genes $(1,026)$ randomly selected from the complete HUGO gene set $(25,254)$. The PPI network of the ROSdiabetes targets was significantly different from the randomly generated networks indicating their strong association with the topic "ROS and Diabetes". Table 2 demonstrates that the mean number of targets with any PPI interaction in the randomly generated target sets was 528.9 (approximately $52 \%$ of 1,026 targets), while the number of targets with any PPI interaction in the ROS-diabetes target was 983 (96\%). The number of targets interacting with each other was also significantly different between the random networks $($ mean $=155.4)$ and the ROS-diabetes network (mean $=879$ ). Figure 1 illustrates the distributions of these measurements from the 100 random networks with the ROS-diabetes set depicted as a red vertical line. It is obvious that the PPI network of the ROS-diabetes targets is significantly different from the random networks.

\section{Functional enrichment analyses of the ROS-diabetes targets}

Functional enrichment analyses of the 1,026 ROS-diabetes targets were performed to identify over-represented biological functions of the ROS-diabetes targets. After Benjamini-Hochberg correction, a total of 189 molecular functions, 450 biological processes, 73 cellular components and 341 pathways were significantly enriched in the ROS-diabetes targets when compared against all the HUGO genes (see Additional Files 3, 4, 5 and 6 for the full lists). Table 3 lists the top 3 most over-represented GO terms and pathways ranked by p-values of Fisher's exact test: e.g., apoptosis, oxidoreductase activity and insulin signaling pathway.

\section{Identification of over-represented ROS-diabetes targets}

To identify the ROS-diabetes targets highly overrepresented in ROS-diabetes literature, three sets of background corpora of the same size ( $\mathrm{n}=1,154$ documents) were generated using the same journal, volume and issue approach. The overlap among the three background sets in terms of documents and identified targets are illustrated in Figure 2. Approximately 90\% of the selected background documents were unique to the individual set, while $50 \%$ of the identified targets were identified in at least one of the three background document sets. The frequencies of the identified targets were compared among the background sets for significant differences. None of the targets had a BH adjusted p-value

Table 2 Summary of 100 randomly generated PPI networks

\begin{tabular}{|c|c|c|c|c|}
\hline & $\begin{array}{r}\text { \# of targets } \\
\text { with any } \\
\text { interaction }\end{array}$ & $\begin{array}{r}\text { \# of targets } \\
\text { interacting with } \\
\text { each other }\end{array}$ & $\begin{array}{r}\text { \# of direct } \\
\text { interactions } \\
\text { among targets }\end{array}$ & Max degree* \\
\hline ROS-diabetes Targets & 983 & 879 & 5002 & 173 \\
\hline Mean (100 networks) & 528.9 & 155.4 & 165.4 & 25 \\
\hline STDEV (100 networks) & 16 & 36.2 & 54.2 & 39.7 \\
\hline Z-Score & 28.5 & 20 & 89.2 & 3.7 \\
\hline P-value(Z) & 0 & 0 & 0 & 9.60E-05 \\
\hline T-Statistics & -284.8 & -200 & -891.9 & -37.3 \\
\hline P-value(T) & $4.60 \mathrm{E}-146$ & $6.70 E-131$ & 4.00E-195 & 4.20E-60 \\
\hline
\end{tabular}

* Max degree refers to the number of interactions of the most highly interacting target. 


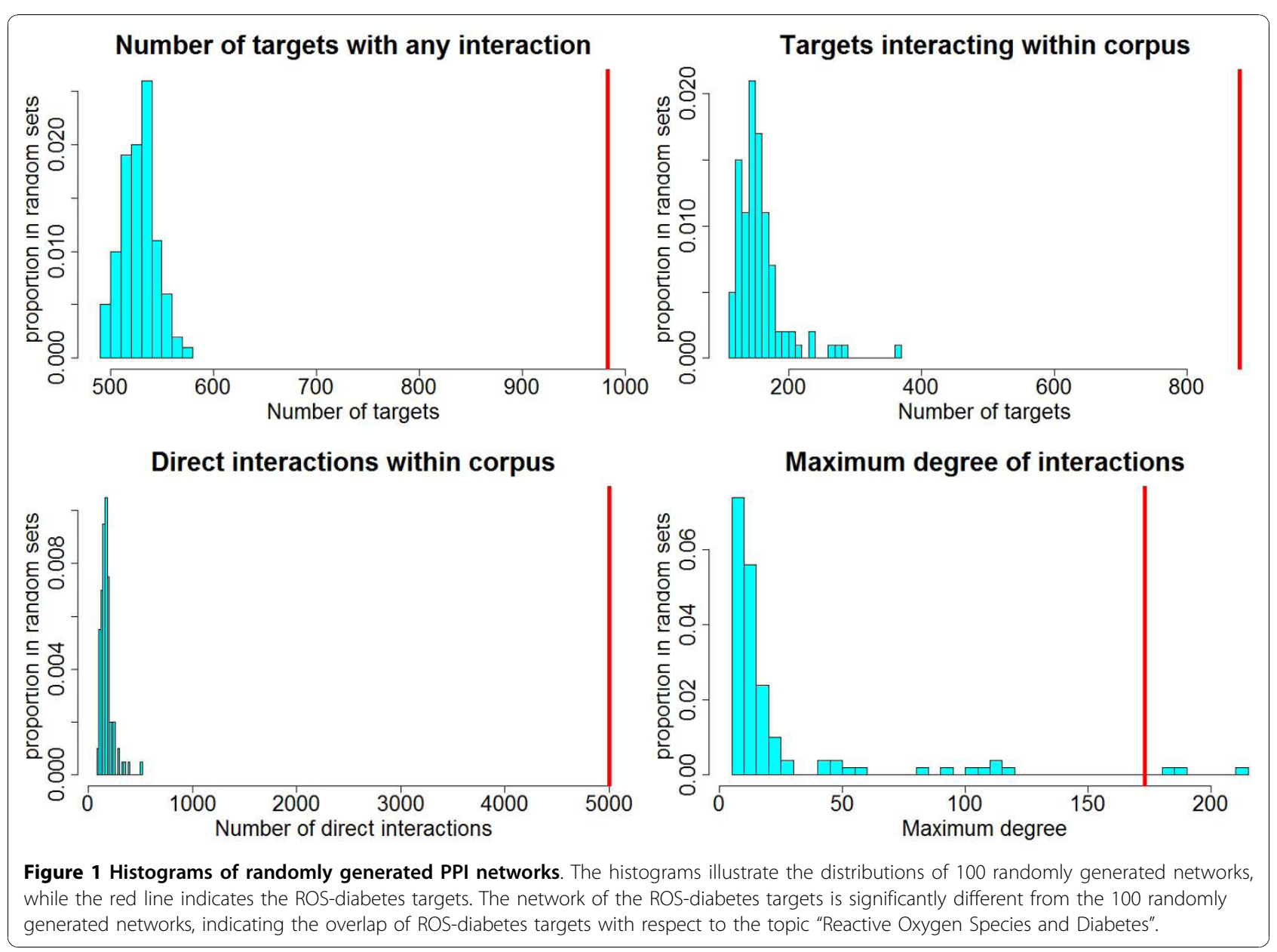

$<0.05$, indicating no significant difference among the targets from the three different background sets (See Additional File 7).

Comparisons of the ROS-diabetes targets against these background sets revealed 53 highly over-represented
ROS-diabetes targets as listed in Table 4 . These 53 targets were significant ( $p$-value $<0.05$ ) against all three background sets and significant following BenjaminiHochberg multiple testing correction (BH adjusted $\mathrm{p}$-value $<0.05)$ against at least two of the three

Table 3 Enriched functions of 1,026 ROS-diabetes targets

\begin{tabular}{|c|c|c|c|c|}
\hline Category & Term & \#target & p-value & Fold \\
\hline \multirow[t]{3}{*}{ Biological Processes GO } & metabolic process & 113 & $3.40 \mathrm{E}-26$ & 3.3 \\
\hline & protein amino acid phosphorylation & 98 & $2.90 \mathrm{E}-24$ & 3.5 \\
\hline & response to hypoxia & 36 & $8.80 \mathrm{E}-24$ & 12 \\
\hline \multirow[t]{3}{*}{ Molecular Functions GO } & protein binding & 514 & $2.80 \mathrm{E}-71$ & 2.1 \\
\hline & oxidoreductase activity & 103 & $1.50 \mathrm{E}-31$ & 4.2 \\
\hline & transferase activity & 148 & $1.70 \mathrm{E}-26$ & 2.7 \\
\hline \multirow[t]{3}{*}{ Cellular Components GO } & cytoplasm & 381 & $1.50 \mathrm{E}-57$ & 2.3 \\
\hline & extracellular region & 220 & $9.10 \mathrm{E}-44$ & 2.9 \\
\hline & mitochondrion & 154 & $6.30 \mathrm{E}-43$ & 3.9 \\
\hline \multirow[t]{3}{*}{ Pathway } & Focal adhesion & 75 & $2.40 \mathrm{E}-42$ & 9.4 \\
\hline & Apoptosis & 49 & $6.70 \mathrm{E}-35$ & 14.5 \\
\hline & MAPK signaling pathway & 73 & $4.30 \mathrm{E}-34$ & 6.9 \\
\hline
\end{tabular}

'\#target' refers to the number of ROS-diabetes targets with each biological function with Benjamini-Hochberg adjusted p-values. Fold is the ratio of targets from the ROS-diabetes set to the complete HUGO gene set. 


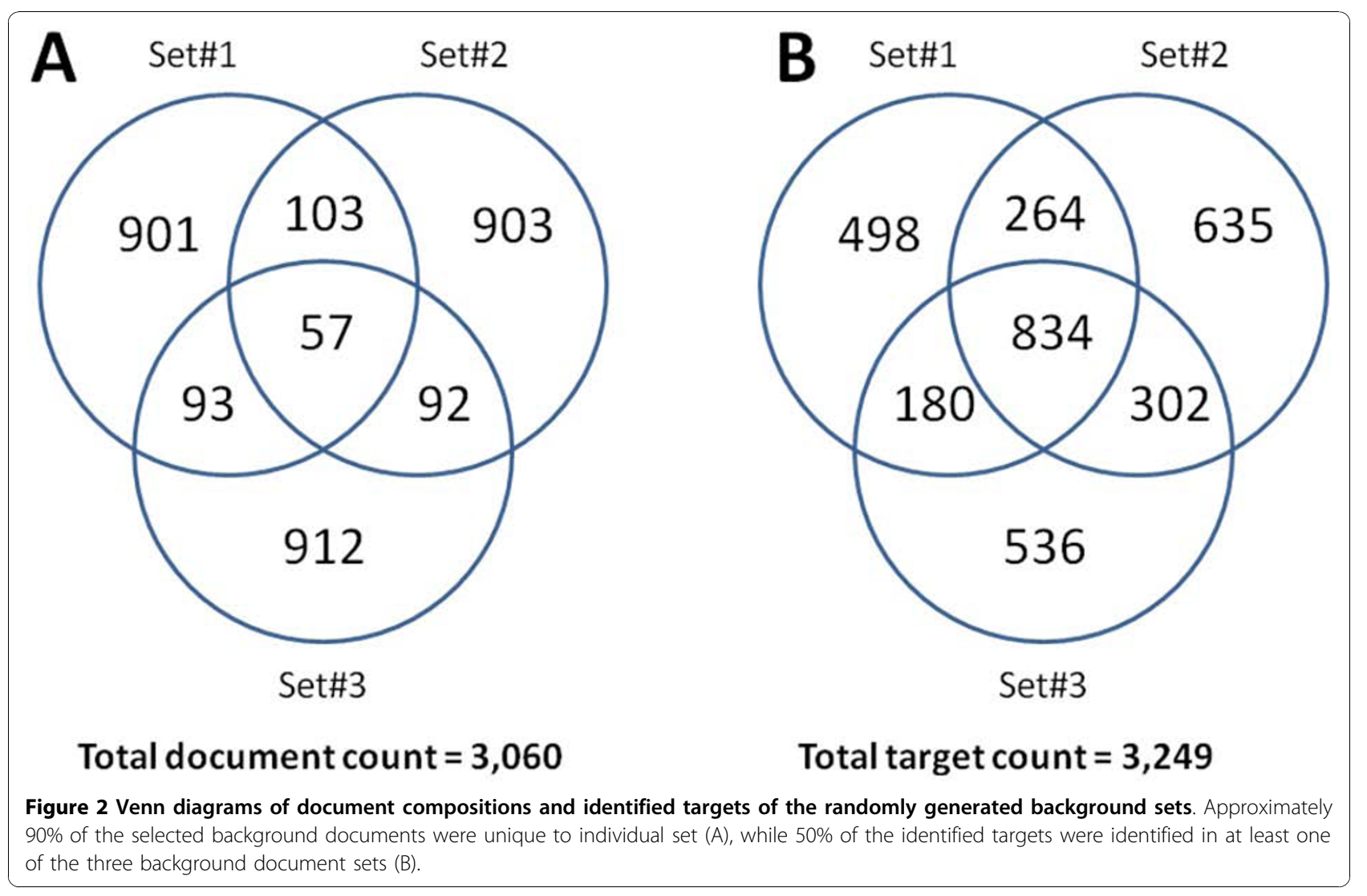

background sets. SOD1 was the most over-represented in the ROS-diabetes targets.

\section{Functional enrichment analyses of the over-represented ROS-diabetes targets}

Functional enrichment analyses of the 53 ROS-diabetes targets were performed to identify over-represented biological functions. Following Benjamini-Hochberg correction, a total of 65 molecular functions, 209 biological processes, 26 cellular components and 108 pathways were significantly over-represented when compared against all the HUGO genes (see Additional Files 8, 9, 10 and 11 for the full lists). Table 5 shows the top 3 most significantly over-represented GO terms and pathways ranked by p-values of Fisher's exact test. GO terms related to oxidative stress such as "superoxide metabolic process", "superoxide release", "electron carrier activity" and "mitochondrion" were highly over-represented in the 53 ROS-diabetes targets.

\section{Gene expression change in diabetes}

Two groups of DBA/2J mice exhibited significantly different levels of glycosylated hemoglobin (\%GHb). The mean \pm SEM were $6.2 \pm 0.3$ for the non-diabetic control group and for $14.0 \pm 0.8$ for the diabetic group ( $\mathrm{p}$-value $<0.001)$, indicative of prolonged hyperglycemia in the diabetic group [26]. DRG were harvested from these animals for gene expression assays. Nine genes were selected from the top ranked ROS-diabetes targets: superoxide dismutase 1 (Sod1), catalase (Cat), xanthine dehydrogenase $(\mathrm{Xdh})$, protein kinase $\mathrm{C}$ alpha (Prkca), neutrophil cytosolic factor 1 (Ncf1), nitric oxide synthase 3 (Nos3), superoxide dismutase 2 ( $\operatorname{Sod} 2)$, cytochrome b-245 alpha (Cyba), and nitric oxide synthase 1 (Nos1). Eight genes exhibited differential expression between diabetic and non-diabetic mice (p-value < 0.05) as shown in Figure 3. Cat, Sod1, Sod2, Prkca, and Nos1 expression levels were decreased, while Ncf1, Xdh, and Cyba expression levels were increased in diabetes.

\section{Discussion}

Reactive oxygen species (ROS) are products of normal energy metabolism and play important roles in many other biological processes such as the immune response and signaling cascades [4-6]. As mediators of cellular damage, ROS are implicated in pathogenesis of multiple diseases including diabetic complications [27-30]. With the aid of literature mining technology, we collected 1,026 possible ROS-related targets from a set of biomedical literature indexed with both ROS and diabetes.

Fifty-three targets were significantly over-represented in the ROS-diabetes papers when compared against 
Table 453 targets over-represented in ROS-diabetes literature

\begin{tabular}{|c|c|c|c|c|c|c|c|}
\hline Rank & Symbol & HUGO_ID & Name & \#Paper & BG \#1 & BG \#2 & BG \#3 \\
\hline 1 & SOD1 & 11179 & $\begin{array}{l}\text { superoxide dismutase 1, soluble (amyotrophic } \\
\text { lateral sclerosis } 1 \text { (adult)) }\end{array}$ & 368 & $3.1 \mathrm{E}-84$ & $2.0 \mathrm{E}-78$ & $2.0 \mathrm{E}-78$ \\
\hline 2 & CAT & 1516 & catalase & 241 & $2.1 \mathrm{E}-50$ & $3.9 \mathrm{E}-44$ & $3.9 \mathrm{E}-44$ \\
\hline 3 & NOX5 & 14874 & NADPH oxidase, EF-hand calcium binding domain 5 & 177 & $3.1 \mathrm{E}-42$ & $3.6 \mathrm{E}-39$ & $2.1 \mathrm{E}-37$ \\
\hline 4 & INS & 6081 & insulin & 503 & $5.9 \mathrm{E}-41$ & $2.0 \mathrm{E}-43$ & $2.3 \mathrm{E}-39$ \\
\hline 5 & $\mathrm{XDH}$ & 12805 & xanthine dehydrogenase & 133 & $1.5 \mathrm{E}-30$ & $1.2 \mathrm{E}-28$ & $8.8 \mathrm{E}-28$ \\
\hline 6 & PRKCA & 9393 & protein kinase $C$, alpha & 194 & $7.1 \mathrm{E}-23$ & $6.4 \mathrm{E}-26$ & $8.9 \mathrm{E}-24$ \\
\hline 7 & NCF1 & 7660 & $\begin{array}{l}\text { neutrophil cytosolic factor 1, (chronic } \\
\text { granulomatous disease, autosomal 1) }\end{array}$ & 72 & $7.6 \mathrm{E}-19$ & 7.7E-16 & $8.7 \mathrm{E}-16$ \\
\hline 8 & NOS3 & 7876 & nitric oxide synthase 3 (endothelial cell) & 115 & $1.6 \mathrm{E}-18$ & $3.9 \mathrm{E}-16$ & 7.6E-18 \\
\hline 9 & SOD2 & 11180 & superoxide dismutase 2 , mitochondrial & 85 & $2.1 \mathrm{E}-18$ & 7.7E-16 & $3.8 \mathrm{E}-15$ \\
\hline 10 & CYBA & 2577 & cytochrome b-245, alpha polypeptide & 69 & $4.2 \mathrm{E}-17$ & $5.0 \mathrm{E}-13$ & $6.9 \mathrm{E}-14$ \\
\hline 11 & NOS2A & 7873 & nitric oxide synthase 2A (inducible, hepatocytes) & 144 & $3.9 \mathrm{E}-16$ & $5.2 \mathrm{E}-12$ & 4.5E-14 \\
\hline 12 & AGT & 333 & angiotensinogen (serpin peptidase inhibitor, clade A, member 8) & 131 & $1.8 \mathrm{E}-14$ & $1.4 \mathrm{E}-09$ & $3.5 \mathrm{E}-08$ \\
\hline 13 & AKR1B1 & 381 & aldo-keto reductase family 1 , member B1 (aldose reductase) & 61 & $8.0 \mathrm{E}-13$ & $9.5 \mathrm{E}-13$ & $3.6 \mathrm{E}-11$ \\
\hline 14 & CYBB & 2578 & cytochrome b-245, beta polypeptide (chronic granulomatous disease) & 49 & 4.0E-12 & 2.6E-09 & $5.8 \mathrm{E}-11$ \\
\hline 15 & NOS1 & 7872 & nitric oxide synthase 1 (neuronal) & 82 & $4.9 \mathrm{E}-12$ & 3.7E-10 & 4.7E-09 \\
\hline 16 & NCF2 & 7661 & $\begin{array}{l}\text { neutrophil cytosolic factor } 2 \text { ( } 65 \mathrm{kDa} \text {, chronic } \\
\text { granulomatous disease, autosomal 2) }\end{array}$ & 50 & $2.4 \mathrm{E}-11$ & $1.5 \mathrm{E}-09$ & $3.8 \mathrm{E}-08$ \\
\hline 17 & CYCS & 19986 & cytochrome c, somatic & 81 & 8.7E-10 & $2.2 \mathrm{E}-10$ & 2.1E-10 \\
\hline 18 & $\mathrm{HBB}$ & 4827 & hemoglobin, beta & 101 & $1.4 \mathrm{E}-08$ & $5.9 \mathrm{E}-10$ & $2.2 \mathrm{E}-08$ \\
\hline 19 & GSR & 4623 & glutathione reductase & 61 & $1.4 \mathrm{E}-08$ & $4.8 \mathrm{E}-08$ & 4.8E-08 \\
\hline 20 & UCP1 & 12517 & uncoupling protein 1 (mitochondrial, proton carrier) & 38 & 4.1E-07 & 2.1E-06 & 9.7E-06 \\
\hline 21 & NOX4 & 7891 & NADPH oxidase 4 & 31 & $6.2 \mathrm{E}-07$ & $2.3 \mathrm{E}-04$ & 2.7E-05 \\
\hline 22 & PARP1 & 270 & poly (ADP-ribose) polymerase family, member 1 & 37 & $7.1 \mathrm{E}-07$ & $1.1 \mathrm{E}-07$ & $5.3 \mathrm{E}-05$ \\
\hline 23 & UCP2 & 12518 & uncoupling protein 2 (mitochondrial, proton carrier) & 34 & $7.0 \mathrm{E}-07$ & 4.5E-06 & 2.1E-05 \\
\hline 24 & HBA1 & 4823 & hemoglobin, alpha 1 & 30 & $1.1 \mathrm{E}-06$ & $1.2 \mathrm{E}-06$ & $9.3 \mathrm{E}-06$ \\
\hline 25 & ALB & 399 & albumin & 179 & $7.0 \mathrm{E}-06$ & $4.9 \mathrm{E}-06$ & 1.7E-06 \\
\hline 26 & NOX1 & 7889 & NADPH oxidase 1 & 30 & $8.2 \mathrm{E}-06$ & $8.6 \mathrm{E}-06$ & 9.7E-06 \\
\hline 27 & NFKB1 & 7794 & $\begin{array}{l}\text { nuclear factor of kappa light polypeptide gene } \\
\text { enhancer in B-cells } 1 \text { ( } 105)\end{array}$ & 90 & $9.4 \mathrm{E}-06$ & $1.2 \mathrm{E}-04$ & 4.5E-04 \\
\hline 28 & VEGFA & 12680 & vascular endothelial growth factor $\mathrm{A}$ & 57 & $2.6 \mathrm{E}-04$ & $1.9 \mathrm{E}-04$ & 4.1E-03 \\
\hline 29 & SOD3 & 11181 & superoxide dismutase 3, extracellular & 18 & $2.5 \mathrm{E}-04$ & $8.1 \mathrm{E}-02$ & $3.4 \mathrm{E}-02$ \\
\hline 30 & REN & 9958 & renin & 51 & $3.6 \mathrm{E}-04$ & $2.2 \mathrm{E}-02$ & $7.2 \mathrm{E}-02$ \\
\hline 31 & MPO & 7218 & myeloperoxidase & 28 & $5.7 \mathrm{E}-04$ & 2.4E-01 & $5.1 \mathrm{E}-02$ \\
\hline 32 & SORD & 11184 & sorbitol dehydrogenase & 15 & $1.8 \mathrm{E}-03$ & $1.9 \mathrm{E}-03$ & $1.8 \mathrm{E}-03$ \\
\hline 33 & COL4A1 & 2202 & collagen, type IV, alpha 1 & 15 & $1.8 \mathrm{E}-03$ & $1.3 \mathrm{E}-02$ & $1.8 \mathrm{E}-03$ \\
\hline 34 & TGFA & 11765 & transforming growth factor, alpha & 46 & $2.1 \mathrm{E}-03$ & $3.5 \mathrm{E}-02$ & $3.5 \mathrm{E}-04$ \\
\hline 35 & ACE & 2707 & angiotensin I converting enzyme (peptidyl-dipeptidase A) 1 & 69 & $3.8 \mathrm{E}-03$ & $1.1 \mathrm{E}-02$ & 1.1. $\mathrm{E}-02$ \\
\hline 36 & AGTR1 & 336 & angiotensin II receptor, type 1 & 36 & 3.7E-03 & $4.9 \mathrm{E}-02$ & $1.8 \mathrm{E}-03$ \\
\hline 37 & G6PD & 4057 & glucose-6-phosphate dehydrogenase & 19 & $5.6 \mathrm{E}-03$ & 3.7E-01 & 2.1E-01 \\
\hline 38 & $\mathrm{CP}$ & 2295 & ceruloplasmin (ferroxidase) & 13 & $6.2 \mathrm{E}-03$ & $3.1 \mathrm{E}-01$ & $2.9 \mathrm{E}-01$ \\
\hline 39 & NCF4 & 7662 & neutrophil cytosolic factor $4,40 \mathrm{kDa}$ & 16 & $6.7 \mathrm{E}-03$ & $9.9 \mathrm{E}-04$ & $9.9 \mathrm{E}-04$ \\
\hline 40 & MT-CYB & 7427 & mitochondrially encoded cytochrome b & 15 & $1.3 \mathrm{E}-02$ & $1.3 \mathrm{E}-02$ & $1.3 \mathrm{E}-01$ \\
\hline 41 & DUOX1 & 3062 & dual oxidase 1 & 11 & $2.2 \mathrm{E}-02$ & $2.9 \mathrm{E}-01$ & 1.1E-01 \\
\hline 42 & SERPINE1 & 8583 & $\begin{array}{l}\text { serpin peptidase inhibitor, clade } E \text { (nexin, plasminogen } \\
\text { activator inhibitor type 1), member } 1\end{array}$ & 37 & $2.4 \mathrm{E}-02$ & $2.5 \mathrm{E}-02$ & $1.1 \mathrm{E}-03$ \\
\hline 43 & GSTCD & 25806 & glutathione S-transferase, C-terminal domain containing & 37 & $2.4 \mathrm{E}-02$ & $3.8 \mathrm{E}-01$ & $9.1 \mathrm{E}-02$ \\
\hline 44 & COQ7 & 2244 & coenzyme Q7 homolog, ubiquinone (yeast) & 16 & $2.8 \mathrm{E}-02$ & $1.9 \mathrm{E}-01$ & $3.1 \mathrm{E}-02$ \\
\hline 45 & RAC1 & 9801 & $\begin{array}{l}\text { ras-related C3 botulinum toxin substrate } 1 \\
\text { (rho family, small GTP binding protein Rac1) }\end{array}$ & 18 & $3.0 \mathrm{E}-02$ & 4.3E-01 & $7.8 \mathrm{E}-02$ \\
\hline 46 & MAOB & 6834 & monoamine oxidase B & 10 & $3.9 \mathrm{E}-02$ & 4.1E-01 & 4.4E-01 \\
\hline 47 & UCP3 & 12519 & uncoupling protein 3 (mitochondrial, proton carrier) & 17 & 4.7E-02 & 1.7E-02 & $1.8 \mathrm{E}-02$ \\
\hline
\end{tabular}


Table 453 targets over-represented in ROS-diabetes literature (Continued)

\begin{tabular}{|c|c|c|c|c|c|c|c|}
\hline 48 & VCAM1 & 12663 & vascular cell adhesion molecule 1 & 29 & $5.4 \mathrm{E}-02$ & 6.3E-02 & 3.5E-02 \\
\hline 49 & AKT1 & 391 & v-akt murine thymoma viral oncogene homolog 1 & 75 & 5.5E-02 & 4.9E-02 & 6.4E-02 \\
\hline 50 & LEPR & 6554 & leptin receptor & 21 & 8.7E-02 & 3.1E-01 & 1.4E-02 \\
\hline 52 & COL1A1 & 2197 & collagen, type I, alpha 1 & 84 & 8.7E-02 & 2.6E-02 & 1.7E-01 \\
\hline 53 & CCL2 & 10618 & chemokine ( $\mathrm{C}-\mathrm{C}$ motif) ligand 2 & 38 & 2.0E-01 & 4.9E-02 & 1.0E-02 \\
\hline
\end{tabular}

'\#Paper' is the number of papers in ROS-diabetes corpus; BG\#1, BG\#2 and BG\#3 are Benjamini-Hochberg adjusted p-values between ROS-diabetes targets and background sets.

three background sets. Depending on how the background set is defined, the over-represented targets may vary widely. An ideal background set would be the entire PubMed set; however, this is not possible due to limited access to full texts and intense data processing. An alternative method would be to use only abstracts in PubMed, but this may not fully represent the literature. Using only the abstracts, our target identification method resulted in 21 (39\%) of the 53 key ROS-diabetes targets (Additional File 12), suggesting the benefit of rich information in full text documents. In the present study, background documents were randomly selected from the same journal, volume, and issue of the 1,154 ROS-diabetes documents, which were not indexed with "Reactive Oxygen Species"[MeSH] nor "Diabetes Mellitus"[MeSH]. This approach maintained the background corpora not far from the ROS and diabetes context.

The gene expression levels of nine targets selected from the 53 over-represented ROS-diabetes targets were measured in diabetic and non-diabetic DRG. Our laboratory is particularly interested in deciphering the underlying mechanisms of diabetic neuropathy, a major complication of diabetes. Data published by our laboratory both in vitro and in vivo confirm the negative impact of oxidative stress in complication-prone neuron tissues like DRG $[7,10,18,31]$. In an effort to obtain diabetic neuropathy specific targets, SciMiner was employed to further analyze a subset of the ROS-diabetes papers (data not shown). Nerve growth factor (NGF) was identified as the most over-represented target in this subset when compared to the full ROSdiabetes set; however, NGF did not have statistical significance $(\mathrm{BH}$ adjusted $\mathrm{p}$-value $=0.06)$. The relatively small numbers of papers and associated targets may have contributed to this non-significance. Therefore, the candidate targets for gene expression validation were selected from among the 53 over-represented ROS-diabetes targets derived from the full ROS-diabetes corpus.

Among the tested genes, the expression levels of Cat, Sod1, Sod2, Prkca, and Nos1 were decreased, while the expression levels of Ncf1, Xdh, and Cyba were increased under diabetic conditions. Cat, Sod1, and Sod 2 are responsible for protecting cells from oxidative stress by destroying superoxides and hydrogen peroxides [8-11].
Decreased expression of these genes may result in oxidative stress [32]. Increased expression of Cyba and Ncf1, subunits of superoxide-generating nicotinamide adenine dinucleotide phosphate (NADPH) oxidase complex [30], also supports enhanced oxidative stress. Xdh and its inter-convertible form, Xanthine oxidase (Xod), showed increased activity in various rat tissues under oxidative stress conditions with diabetes [33], and also showed increased expression in diabetic DRG in the current study.

Unlike the above concordant genes, protein kinase $C$ and nitric oxide synthases did not exhibit predicted expression changes in diabetes. Protein kinase $\mathrm{C}$ activates $\mathrm{NADPH}$ oxidase, further promoting oxidative stress in the cell $[34,35]$. Decreased expression of Prkca in our diabetic DRG is not parallel with expression levels of other enzymes expected to increase oxidative stress. Between the two nitric oxide synthases tested in the present study, Nos1 (neuronal) expression was significantly decreased (p-value $<0.001$ ) in diabetes, while Nos3 (endothelial) expression was not significant ( $\mathrm{p}$-value $=0.06)$. The neuronal Nos1 is expected to play a major role in producing nitric oxide, another type of highly reactive free radical. Thus, with some exceptions, the majority of the differentially expressed genes in DRG show parallel results to the known activities of these targets in diabetes, suggesting enhanced oxidative stress in the diabetic DRG.

Assessment of antioxidant enzyme expression in diabetes has yielded a variety of results [36-40] depending upon the duration of diabetes, the tissue studied and other factors. In diabetic mice and rats, it is commonly reported that superoxide dismutases are down-regulated [37-40], where data regarding catalase are variable $[36,40]$. PKC is activated in diabetes, but most papers that examined mRNA demonstrated that its expression is largely unchanged [41].

Among the 53 over-represented ROS-diabetes targets, SOD1 was the most over-represented and was differentially expressed under diabetic and non-diabetic conditions. To the best of our knowledge, no published study has investigated the role of SOD1 in the onset and/or progression of diabetic neuropathy. Mutations of SOD1 have long been associated with the inherited form of 
Table 5 Enriched functions of the $\mathbf{5 3}$ over-represented targets in diabetes

\begin{tabular}{lllll}
\hline Category & Term & \# target & p-value & Fold \\
\hline Biological Processes GO & superoxide metabolic process & 7 & $3.70 \mathrm{E}-15$ & 303 \\
& electron transport & 13 & $1.50 \mathrm{E}-12$ & 16 \\
& superoxide release & $5.20 \mathrm{E}-11$ & 298 \\
\hline Molecular Functions GO & electron carrier activity & $1.80 \mathrm{E}-17$ & 27 \\
& oxidoreductase activity & 15 & $2.20 \mathrm{E}-16$ & 14 \\
& iron ion binding & 18 & $4.20 \mathrm{E}-16$ & 21 \\
\hline Cellular Components GO & mitochondrion & 15 & $9.90 \mathrm{E}-08$ & 6 \\
& extracellular space & 13 & $6.60 \mathrm{E}-07$ & 8 \\
& soluble fraction & 10 & $3.20 \mathrm{E}-06$ & 11 \\
\hline Pathway & Leukocyte transendothelial migration & 7 & $6.40 \mathrm{E}-12$ & 36 \\
& Small cell lung cancer & 7 & $1.00 \mathrm{E}-09$ & 38 \\
\hline
\end{tabular}

'\#target' refers to the number of ROS-diabetes targets with each biological function with Benjamini-Hochberg adjusted p-values. Fold is the ratio of targets from the ROS-diabetes set to the complete HUGO gene set.

amyotrophic lateral sclerosis (ALS) [42] and the theory of oxidative stress-based aging [43]. Early reports indicate that knockout of the SOD1 gene does not affect nervous system development [44], although recovery following injury is slow and incomplete $[45,46]$. With respect to diabetes, SOD1 KO accelerates the development of diabetic nephropathy [47] and cataract formation [48]. Thus, examining the SOD1 KO mouse as a model of diabetic neuropathy would be a reasonable follow-up study.

One limitation of the current approach using literature mining technology is incorrect or missed identification of the mentioned targets within the literature. Based on a performance evaluation using a standard text set BioCreAtIvE (Critical Assessment of Information Extraction systems in Biology) version 2 [49], SciMiner achieved $87.1 \%$ recall (percentage identification of targets in the given text), $71.3 \%$ precision (percentage accuracy of identified target) and $75.8 \% \mathrm{~F}$-measure (harmonious average of recall and precision $=(2 \times$ recall $\times$ precision $) /($ recall + precision $))$ before manual revision [14]. In order to improve the accuracy of SciMiner's results, each target was manually reviewed and corrected by checking the sentences in which each target was identified. Approximately, 120 targets $(\sim 10 \%$ of the initially identified targets from the ROS-diabetes papers) were removed during the manual review process. The overall accuracy is expected to improve through the review process; however, the review process did not address targets missed by SciMiner, since we did not thoroughly review individual papers. Instead, 5 missed targets, whose associations with ROS-diabetes literature were available in the NCBI Gene2PubMed database, were added to the final ROS-diabetes target list (Additional File 2).

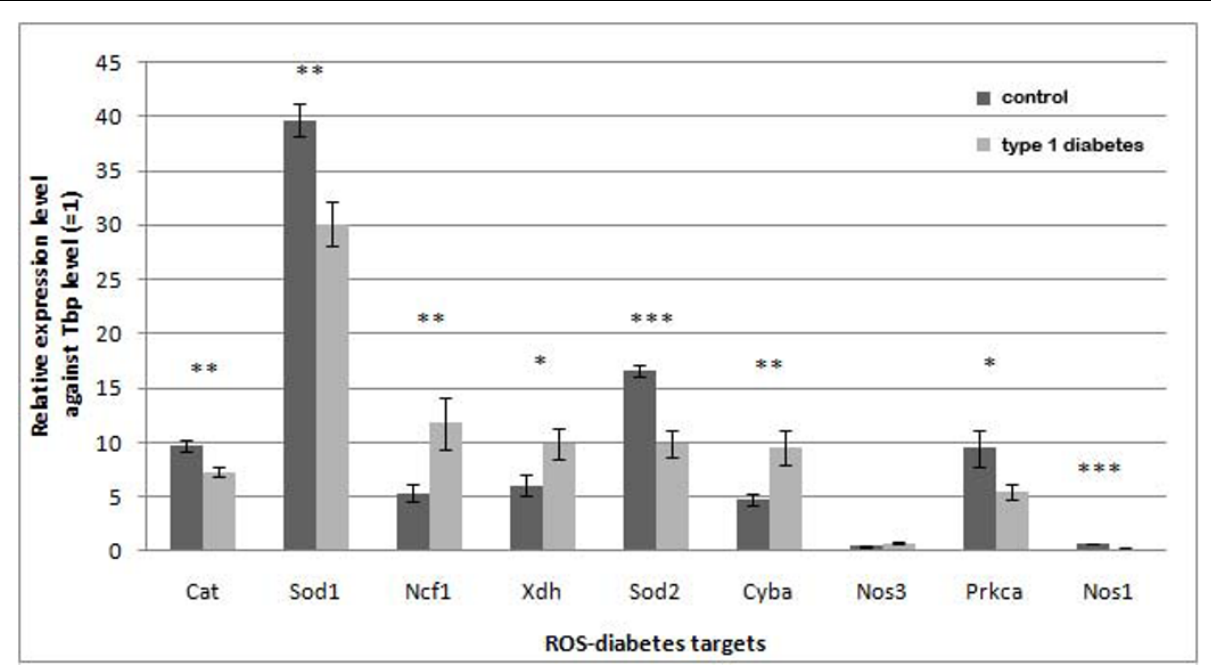

Figure 3 Gene expression levels of selected ROS-diabetes targets in DRG examined by real-time RT-PCR. Expression levels are relative to Tbp, an internal control (error bar $=\operatorname{SEM})\left({ }^{*}, \mathrm{p}<0.05{ }^{* *}, \mathrm{p}<0.01 ;{ }^{* *}, \mathrm{p}<0.001\right)$. Eight (Cat, Sod1, Ncf1, Xdh, Sod2, Cyba, Prkca, and Nos1) out of the nine selected ROS-diabetes genes were significantly regulated by diabetes. 


\section{Conclusions}

The present approach enabled us to collect a comprehensive list of ROS and diabetes related targets and led us to confirm the biological relevance to diabetic neuropathy of the selected ROS-diabetes targets. Using SciMiner to identify significantly enriched targets is applicable to other disease topics of interest by providing a more focused subset of literature for review and by highlighting targets common to multiple manuscripts.

\section{Additional material}

Additional file 1: The list of 90 genes from the NCBI Gene2PubMed
database for the ROS-Diabetes literature (1,154 papers).
Additional file 2: The list of 1,026 ROS-Diabetes targets.
Additional file 3: The enriched Molecular Functions Gene Ontology
Terms in the 1,026 ROS-Diabetes targets.

Additional file 4: The enriched Biological Processes Gene Ontology Terms in the 1,026 ROS-Diabetes targets.

Additional file 5: The enriched Cellular Components Gene Ontology Terms in the 1,026 ROS-Diabetes targets.

Additional file 6: The enriched pathways in the 1,026 ROS-Diabetes targets.

Additional file 7: Comparisons of target frequencies among three background sets.

Additional file 8: The enriched Molecular Functions Gene Ontology Terms in the Over-represented 53 ROS-Diabetes targets.

Additional file 9: The enriched Biological Processes Gene Ontology Terms in the Over-represented 53 ROS-Diabetes targets.

Additional file 10: The enriched Cellular Components Gene Ontology Terms in the Over-represented 53 ROS-Diabetes targets. Additional file 11: The enriched pathways in the Over-represented 53 ROS-Diabetes targets.

Additional file 12: The Key 53 ROS-Diabetes Targets Identifiable Using Only the Abstracts.

\section{List of abbreviations}

NAD(P): nicotinamide adenine dinucleotide (phosphate); AGE: advanced glycoation end-products; PKC: protein kinase C; ROS: reactive oxygen species; MAPK: mitogen-activated protein kinase; JNK: c-Jun amino-terminal kinase; SOD1: superoxide dismutase 1; SOD2: superoxide dismutase 2; CAT: catalase; XDH: xanthine dehydrogenase; NCF1: neutrophil cytosolic factor 1 ; NOS3: nitric oxide synthase 3; CYBA: cytochrome b-245 alpha; NOS1: nitric oxide synthase 1; ALS: amyotrophic lateral sclerosis; BioCreAtlvE: Critical Assessment of Information Extraction systems in Biology; MiMl: Michigan Molecular Interactions; KEGG: Kyoto Encyclopedia of Genes and Genomes; STZ: streptozotocin; GO: gene ontology; DRG: dorsal root ganglia; CNS: central nervous system; HGNC: HUGO (human genome organization) nomenclature committee; PPI: protein-protein interaction; SEM: standard error mean.

\section{Acknowledgements}

The authors acknowledge the expertise of Mr. John Hayes, Dr. Sang Su Oh and Ms. Carey Backus with regard to animal models of diabetes and neuropathy phenotyping methods.

Funding: This work was supported by National Institutes of Health (R01LM008106 to DJS, U54-DA021519 (NCIBI) to DJS, HVJ, and ELF); Animal Models of Diabetic Complications Consortium (AMDCC U01-DK076160) to ELF; Program for Neurology Research and Discovery, and a Rackham Graduate School Predoctoral Fellowship (to JH).

\section{Author details}

'Bioinformatics Program, University of Michigan, Ann Arbor, Ml 48109, USA. ${ }^{2}$ Department of Neurology, University of Michigan, Ann Arbor, Ml 48109, USA. ${ }^{3}$ National Center for Integrative Biomedical Informatics, University of Michigan, Ann Arbor, Ml 48109, USA. ${ }^{4}$ Department of Molecular, Microbial and Structural Biology, University of Connecticut Health Center, Farmington, CT 06030, USA. ${ }^{5}$ School of Health Information Science, University of Texas, Houston, TX 77030, USA.

\section{Authors' contributions}

$\mathrm{JH}$ participated in the study design, performed the literature mining and functional enrichment analyses, and drafted the manuscript. KAS participated in the study design and drafted the manuscript. ADS participated in the statistical analysis. YH carried out the quantitative RT-PCR assay. MP participated in the manuscript revision. DJS participated in the study design and manuscript revision. HVJ participated in the study design and manuscript revision. ELF participated in the study design and manuscript revision. All authors read and approved the final manuscript.

\section{Competing interests}

The authors declare that they have no competing interests.

Received: 6 May 2010 Accepted: 27 October 2010

Published: 27 October 2010

\section{References}

1. American-Diabetes-Association: Economic costs of diabetes in the U.S. In 2007. Diabetes Care 2008, 31(3):596-615

2. Edwards JL, Vincent AM, Cheng HT, Feldman EL: Diabetic neuropathy: mechanisms to management. Pharmacol Ther 2008, 120(1):1-34.

3. Folli F, Guzzi V, Perego L, Coletta DK, Finzi G, Placidi C, La Rosa S, Capella C, Socci C, Lauro D, Tripathy D, Jenkinson C, Paroni R, Orsenigo E, Cighetti G, Gregorini L, Staudacher C, Secchi A, Bachi A, Brownlee M, Fiorina P. Proteomics reveals novel oxidative and glycolytic mechanisms in type 1 diabetic patients' skin which are normalized by kidney-pancreas transplantation. PLoS One 2010, 5(3):e9923.

4. Erol A: Insulin resistance is an evolutionarily conserved physiological mechanism at the cellular level for protection against increased oxidative stress. Bioessays 2007, 29(8):811-818.

5. Pan JS, Hong MZ, Ren JL: Reactive oxygen species: a double-edged sword in oncogenesis. World J Gastroenterol 2009, 15(14):1702-1707.

6. Sarsour EH, Kumar MG, Chaudhuri L, Kalen AL, Goswami PC: Redox control of the cell cycle in health and disease. Antioxid Redox Signal 2009, 11(12):2985-3011.

7. Vincent AM, Russell JW, Low P, Feldman EL: Oxidative stress in the pathogenesis of diabetic neuropathy. Endocr Rev 2004, 25(4):612-628.

8. Schwedhelm E, Maas R, Troost R, Boger RH: Clinical pharmacokinetics of antioxidants and their impact on systemic oxidative stress. Clin Pharmacokinet 2003, 42(5):437-459.

9. Shaw PJ: Molecular and cellular pathways of neurodegeneration in motor neurone disease. J Neurol Neurosurg Psychiatry 2005, 76(8):1046-1057.

10. Vincent AM, Kato K, McLean LL, Soules ME, Feldman EL: Sensory neurons and schwann cells respond to oxidative stress by increasing antioxidant defense mechanisms. Antioxid Redox Signal 2009, 11(3):425-438.

11. Vincent AM, Feldman EL: New insights into the mechanisms of diabetic neuropathy. Rev Endocr Metab Disord 2004, 5(3):227-236.

12. Brownlee M: Biochemistry and molecular cell biology of diabetic complications. Nature 2001, 414(6865):813-820.

13. Feldman EL: Oxidative stress and diabetic neuropathy: a new understanding of an old problem. J Clin Invest 2003, 111(4):431-433.

14. Hur J, Schuyler AD, States DJ, Feldman EL: SciMiner: web-based literature mining tool for target identification and functional enrichment analysis. Bioinformatics 2009, 25(6):838-840.

15. Rebholz-Schuhmann D, Kirsch H, Arregui M, Gaudan S, Riethoven M, Stoehr P: EBIMed-text crunching to gather facts for proteins from Medline. Bioinformatics 2007, 23(2):e237-244.

16. Plake C, Schiemann T, Pankalla M, Hakenberg J, Leser U: AliBaba: PubMed as a graph. Bioinformatics 2006, 22(19):2444-2445. 
17. Cheng D, Knox C, Young N, Stothard P, Damaraju S, Wishart DS: PolySearch: a web-based text mining system for extracting relationships between human diseases, genes, mutations, drugs and metabolites. Nucleic Acids Res 2008, 36 Web Server: W399-405.

18. Russell JW, Berent-Spillson A, Vincent AM, Freimann CL, Sullivan KA, Feldman EL: Oxidative injury and neuropathy in diabetes and impaired glucose tolerance. Neurobiol Dis 2008, 30(3):420-429.

19. Jimenez-Andrade JM, Herrera MB, Ghilardi JR, Vardanyan M, Melemedjian OK, Mantyh PW: Vascularization of the dorsal root ganglia and peripheral nerve of the mouse: implications for chemical-induced peripheral sensory neuropathies. Mol Pain 2008, 4:10.

20. Tarcea VG, Weymouth T, Ade A, Bookvich A, Gao J, Mahavisno V, Wright Z, Chapman A, Jayapandian M, Ozgur A, Tian Y, Cavalcoli J, Mirel B, Patel J, Radev D, Athey B, States D, Jagadish HV: Michigan molecular interactions r2: from interacting proteins to pathways. Nucleic Acids Res 2009, , 37 Database: D642-646.

21. Ashburner M, Ball CA, Blake JA, Botstein D, Butler H, Cherry JM, Davis AP, Dolinski K, Dwight SS, Eppig JT, Harris MA, Hill DP, Issel-Tarver L, Kasarskis A, Lewis S, Matese JC, Richardson JE, Ringwald M, Rubin GM, Sherlock G: Gene ontology: tool for the unification of biology. The Gene Ontology Consortium. Nat Genet 2000, 25(1):25-29.

22. Kanehisa M, Goto S: KEGG: kyoto encyclopedia of genes and genomes. Nucleic Acids Res 2000, 28(1):27-30,

23. Vastrik I, D'Eustachio P, Schmidt E, Joshi-Tope G, Gopinath G, Croft D, de Bono B, Gillespie M, Jassal B, Lewis S, Matthews L, Wu G, Birney E, Stein L: Reactome: a knowledge base of biologic pathways and processes. Genome Biol 2007, 8(3):R39.

24. Fisher RA: On the interpretation of $x^{2}$ from contingency tables, and the calculation of P. Journal of the Royal Statistical Society 1922, 85(1):87-94.

25. Benjamini Y, Hochberg Y: Controlling the False Discovery Rate: A Practical and Powerful Approach to Multiple Testing. Journal of the Royal Statistical Society Series B (Methodological) 1995, 57(1):289-300.

26. Sullivan KA, Hayes JM, Wiggin TD, Backus C, Su Oh S, Lentz SI, Brosius F, Feldman EL: Mouse models of diabetic neuropathy. Neurobiol Dis 2007, 28(3):276-285.

27. Figueroa-Romero C, Sadidi M, Feldman EL: Mechanisms of disease: the oxidative stress theory of diabetic neuropathy. Rev Endocr Metab Disord 2008, 9(4):301-314

28. Hakim FA, Pflueger A: Role of oxidative stress in diabetic kidney disease. Med Sci Monit 2010, 16(2):RA37-48.

29. Ilieva $\mathrm{H}$, Polymenidou $\mathrm{M}$, Cleveland DW: Non-cell autonomous toxicity in neurodegenerative disorders: ALS and beyond. J Cell Biol 2009, 187(6):761-772.

30. Lee HB, Yu MR, Yang Y, Jiang Z, Ha H: Reactive oxygen species-regulated signaling pathways in diabetic nephropathy. J Am Soc Nephrol 2003, 14(8 Suppl 3):S241-245.

31. Vincent AM, Hayes JM, McLean LL, Vivekanandan-Giri A, Pennathur S, Feldman EL: Dyslipidemia-induced neuropathy in mice: the role of oxLDL/LOX-1. Diabetes 2009, 58(10):2376-2385.

32. Cetinkalp S, Delen Y, Karadeniz M, Yuce G, Yilmaz C: The effect of 1alpha,25(OH)2D3 vitamin over oxidative stress and biochemical parameters in rats where Type 1 diabetes is formed by streptozotocin. $J$ Diabetes Complications 2009, 23(6):401-408.

33. Aliciguzel Y, Ozen I, Aslan M, Karayalcin U: Activities of xanthine oxidoreductase and antioxidant enzymes in different tissues of diabetic rats. J Lab Clin Med 2003, 142(3):172-177.

34. laccio A, Collinet C, Gesualdi NM, Ammendola R: Protein kinase C-alpha and -delta are required for NADPH oxidase activation in WKYMVmstimulated IMR90 human fibroblasts. Arch Biochem Biophys 2007, 459(2):288-294.

35. Fontayne A, Dang PM, Gougerot-Pocidalo MA, El-Benna J: Phosphorylation of p47phox sites by PKC alpha, beta II, delta, and zeta: effect on binding to p22phox and on NADPH oxidase activation. Biochemistry 2002, 41(24):7743-7750.

36. Bagi Z, Koller A, Kaley G: PPARgamma activation, by reducing oxidative stress, increases NO bioavailability in coronary arterioles of mice with Type 2 diabetes. Am J Physiol Heart Circ Physiol 2004, 286(2):H742-748.

37. Fukuda M, Nakamura T, Kataoka K, Nako H, Tokutomi Y, Dong YF, Ogawa $H$, Kim-Mitsuyama S: Potentiation by candesartan of protective effects of pioglitazone against type 2 diabetic cardiovascular and renal complications in obese mice. J Hypertens 2010, 28(2):340-352.
38. Fukuda M, Nakamura T, Kataoka K, Nako H, Tokutomi Y, Dong YF, Yasuda O, Ogawa H, Kim-Mitsuyama S: Ezetimibe ameliorates cardiovascular complications and hepatic steatosis in obese and type 2 diabetic $\mathrm{db} / \mathrm{db}$ mice. J Pharmacol Exp Ther 2010

39. Ghosh S, Khazaei M, Moien-Afshari F, Ang LS, Granville DJ, Verchere CB, Dunn SR, McCue P, Mizisin A, Sharma K, Laher I: Moderate exercise attenuates caspase-3 activity, oxidative stress, and inhibits progression of diabetic renal disease in db/db mice. Am J Physiol Renal Physiol 2009, 296(4):F700-708

40. Matsunami T, Sato Y, Sato T, Ariga S, Shimomura T, Yukawa M: Oxidative stress and gene expression of antioxidant enzymes in the streptozotocin-induced diabetic rats under hyperbaric oxygen exposure. Int J Clin Exp Pathol 2009, 3(2):177-188.

41. Kameyama N, Arisawa S, Ueyama J, Kagota S, Shinozuka K, Hattori A, Tatsumi Y, Hayashi H, Takagi K, Wakusawa S: Increase in P-glycoprotein accompanied by activation of protein kinase Calpha and NF-kappaB p65 in the livers of rats with streptozotocin-induced diabetes. Biochim Biophys Acta 2008, 1782(5):355-360.

42. Turner BJ, Talbot K: Transgenics, toxicity and therapeutics in rodent models of mutant SOD1-mediated familial ALS. Prog Neurobiol 2008, 85(1):94-134.

43. Perez VI, Bokov A, Van Remmen H, Mele J, Ran Q, kkeno Y, Richardson A: Is the oxidative stress theory of aging dead? Biochim Biophys Acta 2009, 1790(10):1005-1014.

44. Ohlemiller KK, McFadden SL, Ding DL, Flood DG, Reaume AG, Hoffman EK, Scott RW, Wright JS, Putcha GV, Salvi RJ: Targeted deletion of the cytosolic $\mathrm{Cu} / \mathrm{Zn}$-superoxide dismutase gene (Sod1) increases susceptibility to noise-induced hearing loss. Audiol Neurootol 1999, 4(5):237-246.

45. Kawase M, Murakami K, Fujimura M, Morita-Fujimura $Y$, Gasche $Y$, Kondo $T$, Scott RW, Chan PH: Exacerbation of delayed cell injury after transient global ischemia in mutant mice with CuZn superoxide dismutase deficiency. Stroke 1999, 30(9):1962-1968

46. Reaume AG, Elliott JL, Hoffman EK, Kowall NW, Ferrante RJ, Siwek DF, Wilcox HM, Flood DG, Beal MF, Brown RH, Scott RW Jr, Snider WD: Motor neurons in $\mathrm{Cu} / \mathrm{Zn}$ superoxide dismutase-deficient mice develop normally but exhibit enhanced cell death after axonal injury. Nat Genet 1996, 13(1):43-47.

47. DeRubertis FR, Craven PA, Melhem MF: Acceleration of diabetic renal injury in the superoxide dismutase knockout mouse: effects of tempol. Metabolism 2007, 56(9):1256-1264

48. Olofsson EM, Marklund SL, Behndig A: Enhanced diabetes-induced cataract in copper-zinc superoxide dismutase-null mice. Invest Ophthalmol Vis Sci 2009, 50(6):2913-2918.

49. Hirschman L, Yeh A, Blaschke C, Valencia A: Overview of BioCreAtlvE: critical assessment of information extraction for biology. BMC Bioinformatics 2005, 6(Suppl 1):S1.

\section{Pre-publication history}

The pre-publication history for this paper can be accessed here: http://www.biomedcentral.com/1755-8794/3/49/prepub

\section{doi:10.1186/1755-8794-3-49}

Cite this article as: Hur et al:: Literature-based discovery of diabetesand ROS-related targets. BMC Medical Genomics 2010 3:49.

\section{Submit your next manuscript to BioMed Central and take full advantage of:}

- Convenient online submission

- Thorough peer review

- No space constraints or color figure charges

- Immediate publication on acceptance

- Inclusion in PubMed, CAS, Scopus and Google Scholar

- Research which is freely available for redistribution 